\title{
Renewable Energy Distribution in Cooperative Cellular Networks with Energy Harvesting
}

\author{
Navid Reyhanian*, Vahid Shah-Mansouri*, Behrouz Maham*†, and Chau Yuen ${ }^{\ddagger}$ \\ ${ }^{*}$ School of Electrical and Computer Engineering, University of Tehran, Tehran, Iran \\ ${ }^{\dagger}$ Department of Electrical and Electronic Engineering, School of Engineering, Nazarbayev University, Astana, Kazakhstan \\ ${ }^{\ddagger}$ Singapore University of Technology and Design, Singapore \\ Email: \{n.reyhanian, vmansouri, bmaham\}@ut.ac.ir, yuenchau@sutd.edu.sg
}

\begin{abstract}
In this paper, we propose a novel online centralized algorithm for energy cooperation among energy harvesting capable base stations (BSs) in multi-tier cellular networks. BSs are connected to the non-renewable source used by a BS when it cannot harvest sufficient energy to serve its connected users. BSs with the extra harvested energy operate cooperatively and share their surplus energy with BSs that have not harvested sufficient energy. To stimulate BSs with energy deficit to use the shared energy of other BSs, an energy pricing framework is established which results in reducing of the non-renewable energy consumption. We formulate the problem of maximizing the fairness of the renewable energy distribution. The closed-form of energy share given to each BS with energy deficit is found, by which the renewable energy distribution fairness is maximized. Energy is shared by the smart grid. The problem of minimizing the smart grid usage cost for distributing energy is formulated and an online algorithm is proposed to approximate its solution. Simulation results show that the approximate algorithm reduces the non-renewable energy consumption significantly and reduces the cost of smart grid usage near to the optimal solution.
\end{abstract}

\section{INTRODUCTION}

Deploying small cells (e.g., femtocells and picocells) along with traditional macrocells in cellular networks is a promising solution to meet the exploding demand of the capacity and coverage [1]. Depending on their capabilities, small cell base stations (BSs) are classified into different types such as microcells, picocells and femtocells. Various technical challenges raise when such cells are deployed such as resource assignment, power allocation and interference management. Since small cells has short coverage and limited power consumption, one can think of running those devices using battery power. To make the cellular network green, energy harvesting from renewable energy sources for better charging has received attention. Harvesting energy from the sources like solar and wind power is a thriving economical approach for reducing the use of fossil energy resources [2], [3]. In such cases, one need to model both the battery charging behavior as well as user power consumptions.

Energy harvesting receivers are considered in [4] where channel state information is used in order to find an adaptive energy beamforming to supply energy to receivers. The power consumption model of BSs from different types in cellular networks is investigated in [5]. In [5], the relation between the BSs transmit power and the users requested traffic rates is obtained. In [6], a heterogeneous network is studied where BSs in different tiers are self-powered. If a BS has not harvested sufficient energy, it is kept OFF for charging energy, and its connected users are served by neighbouring BSs which are ON. In [7], the authors study a non-cooperative game among storage

This research is partly supported by A*Star SERC project number 14202 00043. units. In order to maximize its utility, each storage unit decides strategically on the amount energy to sell in a local market. The energy exchange is done by the smart grid. The game is guaranteed to have at least one Nash equilibrium. Energy cooperation in energy harvesting networks has been explored recently, for instance, in [8], [9], where harvesting modules share a portion of their harvested energy with other harvesting modules. In [8], the optimal energy cooperation among a source and a relay is found that maximizes the network throughput.

The energy cooperation between two cellular BSs equipped with harvesting and hybrid modules is studied in [9]. In [9], the harvested energy and the demanded energy are considered to be deterministic and the optimal energy cooperation policy of BSs is derived. In [10], the joint energy and communication cooperation approaches for energy cost saving in cellular networks is studied where the smart grid facilitates two-way energy exchange. The joint design and combination of the physical layer technique of the coordinated multi-point (CoMP) with two way energy trading is studied in [11], where BSs are connected to the smart grid. In contrary to these solutions which considers trade with smart grid, in our framework, the harvested energy is not traded between the BS and the grid. We propose a framework for energy exchange among BSs by which one can combat the effect of the intermittent nature of the harvested energy from nonrenewable source. A non-cooperative Stackelberg game between the residential units of energy and the shared facility controller is proposed in [12] to explore how both entities can benefit from their energy trading with each other and the grid. Optimal energy management decisions to minimize the total electricity cost and the operation delay is investigated in [13] where users are connected to smart grid.

In this paper, an online centralized approach for reducing the non-renewable energy consumption in multi-tier cellular networks with energy harvesting capability is studied. We assume that the harvested and the demanded energy from BSs have stochastic nature. BSs with the extra harvested energy operate cooperatively and share their surplus energy with BSs that have not harvested sufficient energy. To stimulate BSs with energy deficit to utilize the harvested energy of other BSs, an energy pricing framework is established resulting in reducing the non-renewable energy consumption. By using the Jain's fairness index, the fairness of the renewable energy distribution is quantified. We formulate the problem of maximizing the fairness of the renewable energy distribution. We derive an equation which shows the share each BS with deficit energy receives such that the fairness between BSs is maximized. Energy is shared by the smart grid. When BSs share their energy using the smart grid, the grid operator charges a cost for such service. This cost increases as the amount of the shared energy and the distance among BSs increases. The problem of minimizing the smart grid 
usage cost for distributing energy is formulated and an online algorithm is proposed to approximate its solution. Simulation results show that the approximate algorithm reduces the nonrenewable energy consumption significantly and reduces the cost of smart grid usage near to the optimal solution. The cost of installing large batteries is removed as the proposed algorithm is applied and the waste of energy in limited batteries reduces.

The rest of this paper is organized as follows. The system model is given in Section II. Our proposed energy distribution frameworks is presented in Section III and performance evaluations are given in Section VI.

\section{SySTEM MODEL}

Consider the downlink of a small cell network that encompasses $K$ tiers of BSs. Assume that BSs operating in tier $k$ are distributed according to a Poisson Points Process (PPP) with density $\lambda_{k}$. Without lack of generality, we assume that the only source of energy consumption in a BS is the energy used to serve the connected users. The downlink transmit power of a BS operating in tier $k$ is $P_{k}$. The distribution of the users follows a PPP with density $\lambda_{u}$ and each user is allowed to connect to only one BS. Tiers are unbiased and a user connects to a BS with the highest average received signal power. As the transmit power and densities of BSs operating in different tiers are not identical, the number of users served by BSs are not equal. The average number of users that a BS of tier $k$ serves is [6]

$$
N_{k}=\frac{P_{c} \lambda_{u} P_{k}^{\frac{2}{\gamma}}}{\sum_{j=1}^{K} \lambda_{j} P_{j}^{2 / \gamma}},
$$

where $\gamma$ is the pass loss exponent considered equal for all the tiers. Moreover, $P_{c}$ is the coverage probability and denotes the portion of the users connected to a BS with SINR above than a threshold. We consider a time-slotted system where a user demands a traffic rate in bits per second at the beginning of each time slot from the connected BS. The demanded rate has a stochastic nature and it is assumed to be constant during a time slot. Let $R_{i, k}^{m}(t)$ and $p_{i, k}^{m}(t)$ respectively denote the demanded rate of the user $m$ connected to the $i^{\text {th }} \mathrm{BS}$ of tier $k$ at time slot $t$ and the power consumption of the BS in Watts to provide the user with its demanded rate. Due to the stochastic nature of demanded rates of connected users, the consumed power at BSs is stochastic. The consumed power to serve each user connected to a BS can be modeled as an arbitrary random process. The number of connected users to the $i^{\text {th }}$ BS operating in tier $k$ at time slot $t$ is denoted by $N_{k}^{i}(t)$. It is modeled as a Poisson random variable [5], where its mean value is given in [1). If $p_{k}^{i}(t)$ denotes the consumed power of the $i^{\text {th }} \mathrm{BS}$ of tier $k$ at time slot $t$, we have $p_{k}^{i}(t)=\sum_{m=1}^{N_{k}^{i}(t)} p_{i, k}^{m}(t)$. Since the consumed power is constant throughout the time slot, the consumed energy is $T p_{k}^{i}(t)$ Joules where $T$ is the time slot duration.

The BSs harvest energy from environment. The amount of the harvested energy of the $i^{\text {th }} \mathrm{BS}$ of tier $k$ at time slot $t$ is denoted by $\mu_{k}^{i}(t)$. It has a stochastic nature. In our model, we have no restriction on the distribution of this variable. A BS stores the harvest energy in its battery and its capacity is limited. We assume that all the BSs of tier $k$ have similar battery capacities of $c_{k}$. The harvested energy is wasted if the battery is full. Let $e_{k}^{i}(t)$ denote the battery level of the $i^{\text {th }} \mathrm{BS}$ of tier $k$ at time slot $t$. As the harvested energy has stochastic nature, it is possible that in some time slots, the harvested energy by a BS is more than the required energy to serve its connected users. On the other hand, in some times lots, the harvested energy is not sufficient to serve its connected users. The BS is compelled to compensate its energy deficit in such cases.

To compensate their energy deficit, BSs are connected to the non-renewable energy source. To reduce the non-renewable energy consumption, we allow a BS with extra harvested energy to share its surplus energy with BSs with energy deficit. The energy exchange between BSs is carried out using the smart grid. A smart grid enables a precise measurement of the electric power by using digital devices which can communicate with each other. When the BSs share their energy using the smart grid, the smart grid operator charges a cost for such service. This cost is an increasing function of the distance as well as the amount of the shared energy [14]. In our model, we assume that distances between BSs are known. It is supposed that the energy transfer from a BS to other BSs is possible. Assume that the $i^{\text {th }}$ BS of tier $k$ shares $E_{S}(t)$ Joules with other BSs or receives $E_{S}(t)$ Joule from other BSs. Its battery level is updated as

$$
\begin{aligned}
& e_{k}^{i}(t+1)= \\
& \min \left\{\max \left\{e_{k}^{i}(t)-T p_{k}^{i}(t), 0\right\}+\mu_{k}^{i}(t) \pm E_{S}(t+1), c_{k}\right\},
\end{aligned}
$$

where $E_{S}(t)$ is added if the $\mathrm{BS}$ receives energy, or it is subtracted if the BS shares its extra stored energy with other BSs. In (2), the maximum stored energy in the battery is equal to the battery capacity. Furthermore, the BS can not use more energy than the stored amount from its battery.

\section{RENEWABLE ENERGy Distribution FramewORKS}

Based on the system model presented in the previous section, we build a framework for energy exchange between BSs. The goal is to reduce the non-renewable energy consumption performed such that the maximum of the renewable energy distribution fairness is attained. Simultaneously, the cost of using the smart grid for renewable energy distribution among BSs with energy deficit is minimized. Consider that the energy consumption by a BS to serve a user costs a known price of $\varphi$ units of money per Joule for the user. Moreover, the used non-renewable energy costs $\zeta$ units of money per Joule for a BS where $\varphi<\zeta$. In order to make the financial transactions, a Credit Clearance Service (CCS) is used [15], [16], where all the BSs have credit accounts with initial fund. We assume that the CCS is also the network control center.

At the beginning of each time slot, all the BSs send a message to the CCS which contains the information of the amount of energy they are willing to share or receive. Based on these information, the BSs are divided into two groups, i.e, BSs with extra harvested energy and BSs with energy deficit. Let $\mathcal{S}$ denote the set of BSs with extra harvested energy, where $n=|\mathcal{S}|$ denotes its cardinality. We denote the $i^{\text {th }} \mathrm{BS}$ in $\mathcal{S}$ by $s_{i}$. The set of BSs with energy deficit is denoted by $\mathcal{B}$ and its cardinality is $n^{\prime}=|\mathcal{B}|$. We denote the $j^{\text {th }} \mathrm{BS}$ in $\mathcal{B}$ by $b_{j}$. We update our notation for the battery level, the number of connected users and the demanded power based on our new definitions as follows. $e_{s_{i}}(t), N_{s_{i}}(t)$ and $p_{s_{i}}(t)$ are used for the BS $s_{i}$ with the extra harvested energy, and $e_{b_{j}}(t), N_{b_{j}}(t)$ and $p_{b_{j}}(t)$ are used for the BS $b_{j}$ with energy deficit, respectively. The cost of transferring a unit of energy from BS $s_{i}$ to $\mathrm{BS} b_{j}$ is captured by an increasing function $\Gamma\left(g_{s_{i}, b_{j}}\right)$ where $g_{s_{i}, b_{j}}$ is the distance between BSs $s_{i}$ and $b_{j}$ in meters. We assume that $\Gamma\left(g_{s_{i}, b_{j}}\right)<\varphi, \forall s_{i} \in \mathcal{S}, \forall b_{j} \in \mathcal{B}$. This inequality is used to show that the non-renewable energy costs more than the shared energy of other BSs, and thus, a BS is motivated to compensate its energy deficit by the extra stored energy of other BSs. Moreover, this inequality ensures that a BS with energy deficit has incentive 
to compensate its energy shortage with extra stored energy of other BSs to serve connected users. The cost of the smart grid usage is paid by the BS receiving the energy (i.e., BS with energy deficit). Let $\rho_{s_{i}}(t)$ denote the amount of extra energy the BS $s_{i}$ is willing to share at time slot $t$. Then, we find

$$
\rho_{s_{i}}(t)=e_{s_{i}}(t)-T p_{s_{i}}(t) \text {. }
$$

The amount of the required energy of the BS $b_{j}$ at time slot $t$ is

$$
\rho_{b_{j}}(t)=\left|e_{b_{j}}(t)-T p_{b_{j}}(t)\right| .
$$

The total extra energy stored in BSs of the network at time slot $t$ is $\sum_{i=1}^{|\mathcal{S}|} \rho_{s_{i}}(t)$. The total needed energy of BSs with energy deficit at time slot $t$ is $\sum_{j=1}^{|\mathcal{B}|} \rho_{b_{j}}(t)$. The utility function of the BS $s_{i}$ at time slot $t$ is

$$
U_{s_{i}}(t)=\varphi T p_{s_{i}}(t) .
$$

The energy share that the $\mathrm{BS} b_{j}$ receives from other $\mathrm{BSs}$ is $\eta_{b_{j}}(t)$. This can be less than the energy that BS $b_{j}$ needs to serve its users. Let $E_{N}(t)$ denote the amount of energy purchased from the non-renewable source in time slot $t$ which is $E_{N}(t)=$ $\rho_{b_{j}}(t)-\eta_{b_{j}}(t)$. The utility function of the BS $b_{j}$ at time slot $t$ is

$$
U_{b_{j}}(t)=\varphi T p_{b_{j}}(t)-\zeta E_{N}(t)-\Gamma\left(g_{s_{i}, b_{j}}\right) \eta_{b_{j}}(t) .
$$

We notice that $\Gamma\left(g_{s_{i}, b_{j}}\right) \eta_{b_{j}}(t)$ is the cost of sharing $\eta_{b_{j}}(t)$ Joule between BSs $s_{i}$ and $b_{j}$ by the smart grid.

\section{A. Fair Renewable Energy Distribution Scheme}

The BSs with extra energy operate cooperatively and share their surplus energy with BSs which are in shortage of energy. As $\varphi<\zeta$ and $\Gamma\left(g_{s_{i}, b_{j}}\right)<\varphi, \forall s_{i} \in \mathcal{S}, \forall b_{j} \in \mathcal{B}$, one can obtain $\Gamma\left(g_{s_{i}, b_{j}}\right)<\zeta, \forall s_{i} \in \mathcal{S}, \forall b_{j} \in \mathcal{B}$. Thus, BS $b_{j}$ has incentive to compensate its energy deficit with extra harvested energy of other BSs rather than using non-renewable energy which is more expensive. Since BSs with energy deficit are willing to compensate all their energy deficit with the extra harvested energy of other BSs and the amount of the extra harvested energy is limited, the extra harvested energy should be distributed among all BSs fairly. As BSs have different harvesting rates, the demanded energy and battery capacities, the equal energy share is not fair. To quantify the fairness in a resource allocation problem, various measures have been proposed [17]-[19]. A well-known approach is the one referred by the Jain's index [19]. The Jain's fairness index for allocation of resources among $n$ agents is

$$
\text { fairness }=\frac{\left(\sum_{i=1}^{n} x_{i}\right)^{2}}{n \sum_{i=1}^{n} x_{i}^{2}},
$$

where $x_{i}$ is the allocated share to agent $i$. The Jain's fairness index is between $\frac{1}{n}$ and one, where one denote the highest fairness and $\frac{1}{n}$ measures the lowest level of fairness [19]. Assume that $a$ resources are divided between $n$ similar agents and each agent's share is $a / n$. By substituting $x_{i}=a / n$ in (7), it is observed that the fairness reaches one. In other words, when the agents are similar, the equal share maximizes the fairness. We propose an approach to maximize the fairness of the renewable energy distribution. Each BS may compensate part of its energy deficit with the expensive non-renewable energy. The ratio of the non-renewable energy consumption by the $\mathrm{BS} b_{j}$ to its required energy is $\left(\rho_{b_{j}}(t)-\eta_{b_{j}}(t)\right) / \rho_{b_{j}}(t)$. The $\mathrm{BS} b_{j}$ is motivated to minimize the consumed non-renewable energy which is equal to minimize this ratio. This is equal to maximizing $\eta_{b_{j}}(t) / \rho_{b_{j}}(t)$, which is the ratio of the received renewable energy to the total needed energy of the BS $b_{j}$ as $\eta_{b_{j}}(t)$ is the only variable.

To obtain a fair energy distribution, we use ratio $\eta_{b_{j}}(t) / \rho_{b_{j}}(t)$ as the notion of the resource share of BS $b_{j}$. We use $\eta_{b_{j}}(t) / \rho_{b_{j}}(t)$ instead of $x_{i}$ in 77 . We try to maximize the Jain's index as

$$
\begin{aligned}
\max _{\boldsymbol{\eta}_{b}(t)} & \frac{\left(\sum_{j=1}^{n^{\prime}} \frac{\eta_{b_{j}}(t)}{\rho_{b_{j}}(t)}\right)^{2}}{n^{\prime} \sum_{j=1}^{n^{\prime}}\left(\frac{\eta_{b_{j}}(t)}{\rho_{b_{j}}(t)}\right)^{2}} \\
\text { s.t. } & \eta_{b_{j}}(t) \leq \rho_{b_{j}}(t), \forall b_{j} \in \mathcal{B}, \\
& \sum_{j=1}^{n^{\prime}} \eta_{b_{j}}(t)=\min \left\{\sum_{i=1}^{n} \rho_{s_{i}}(t), \sum_{j=1}^{n^{\prime}} \rho_{b_{j}}(t)\right\}, \\
& 0 \leq \eta_{b_{j}}(t), \forall b_{j} \in \mathcal{B},
\end{aligned}
$$

where $\boldsymbol{\eta}_{b}(t)=\left[\eta^{b_{1}}(t), \ldots, \eta^{b_{n^{\prime}}}(t)\right]$ is the vector of energy shares. The first constraint in (8) shows that a given energy share to the $\mathrm{BS} b_{j}$ is less than or equal to its needed energy. The second constraint states that the aggregate energy that the BSs with energy deficit receive from renewable energy sources is equal to minimum of the total extra energy in the BSs (i.e., $\sum_{i=1}^{n} \rho_{s_{i}}(t)$ ) and the shortage of energy in that BSs (i.e, $\sum_{j=1}^{n^{\prime}} \rho_{b_{j}}(t)$ ).

To solve the above optimization problem in (8), we investigate the convexity of the objective function and the constraints. The constrains are linear, and therefore, they are affine. It is straightforward to show that the objective function is neither convex nor concave in its domain. The maximum of the objective function is one [19]. Since the objective function is neither convex nor concave, by applying KKT conditions, we can find the local and global extremums or saddle points [20]. The global maximum point of the above optimization, is found by the following theorem.

Theorem 1: The global maximum point of the optimization problem formulated in (8) that satisfies all constraints is

$$
\eta_{b_{j}}^{\star}(t)=\frac{\rho_{b_{j}}(t) \min \left\{\sum_{i=1}^{n} \rho_{s_{i}}(t), \sum_{j=1}^{n^{\prime}} \rho_{b_{j}}(t)\right\}}{\sum_{j=1}^{n^{\prime}} \rho_{b_{j}}(t)}, \forall b_{j} \in \mathcal{B} .
$$

Proof: The proof is given in [21].

It is observed from (9) that the energy deficit of BSs is compensated totally when the total stored energy in BSs of the network is more than the total demanded energy from BSs. Thus, the nonrenewable energy usage is zero in this case. Moreover, when the total stored energy in BSs of the network is less than the total demanded energy from BSs, the entire extra stored energy in the network is used. Next, the renewable energy is distributed such that the total cost of the smart grid usage for sharing energy is minimized. To maintain the fairness maximized, the energy share given to BS $b_{j}$ is $\eta_{b_{j}}^{\star}(t)$. Consider that the amount of the shared energy among BSs $s_{i}$ and $b_{j}$ is $\sigma_{s_{i}, b_{j}}$. We assume that $\boldsymbol{\Sigma}$ is the matrix of energy transfer between BSs. The $i^{\text {th }}$ element of the $j^{\text {th }}$ column of $\Sigma$ is $\sigma_{s_{i}, b_{j}}$. We use the following optimization problem to minimize the smart grid usage cost

$$
\max _{\Sigma} \sum_{i=1}^{n} \sum_{j=1}^{n^{\prime}} \Gamma\left(g_{s_{i}, b_{j}}\right) \sigma_{s_{i}, b_{j}}
$$


TABLE I. SUMMARY OF THE APPROXIMATE ALgORITHM FOR ASSIGNING THE SUPPLY TO THE DEMAND

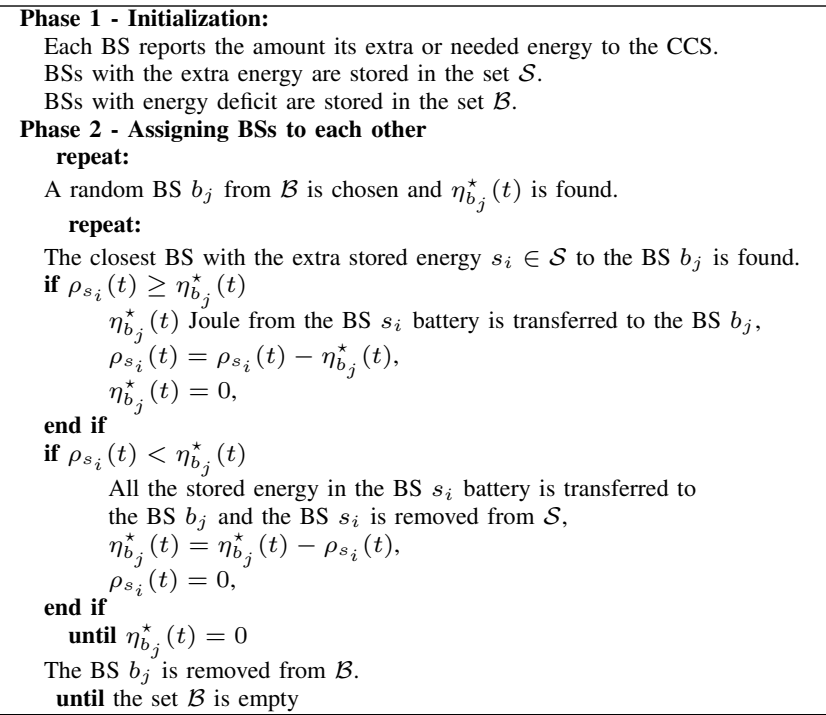

$$
\begin{array}{ll}
\text { s.t. } & \sum_{j=1}^{n^{\prime}} \sigma_{s_{i}, b_{j}} \leq \rho_{s_{i}}(t), \forall s_{i} \in \mathcal{S}, \\
& \sum_{i=1}^{n} \sigma_{s_{i}, b_{j}}=\frac{\rho_{b_{j}}(t) \min \left\{\sum_{i=1}^{n} \rho_{s_{i}}(t), \sum_{j=1}^{n^{\prime}} \rho_{b_{j}}(t)\right\}}{\sum_{j=1}^{n^{\prime}} \rho_{b_{j}}(t)}, \quad \forall b_{j} \in \mathcal{B}, \\
& 0 \leq \sigma_{s_{i}, b_{j}}, \forall s_{i} \in \mathcal{S}, \forall b_{j} \in \mathcal{B} .
\end{array}
$$

The above optimization determines how much energy a BS with energy deficit receives from each BS with the extra stored energy. In the above optimization, $\Gamma\left(g_{s_{i}, b_{j}}\right), \forall s_{i} \in \mathcal{S}, \forall b_{j} \in \mathcal{B}$ is a known constant. The first constraint states that the BSs with extra stored energy can share less or equal to their surplus energy. The second constraint ensures that the fairness is maximized. The last constraint ensures that the shared energy is positive and causal. The optimization problem formulated in $(10)$ is similar to the travelling saleman problem which is demonstrated to be an NPcomplete problem in the class of NP-hard problems [22].

\section{B. Approximate Algorithm for Assigning the Supply to the Demand}

As the optimization problem in 107 is NP-hard, when the number of BSs in the network increases, the computational complexity grows exponentially which is in contrast to the real time energy distribution considered in this paper. To perform the real time energy distribution, an approximate algorithm is proposed which utilizes the concept of the nearest neighbor algorithm. The nearest neighbor algorithm quickly gives an efficient solution [22]. The amount of the energy that BS $b_{j}$ receives from other BSs is $\eta_{b_{j}}^{\star}(t)=\frac{\rho_{b_{j}}(t) \min \left\{\sum_{i=1}^{n} \rho_{s_{i}}(t), \sum_{j=1}^{n^{\prime}} \rho_{b_{j}}(t)\right\}}{\sum_{j=1}^{n^{\prime}} \rho_{b_{j}}(t)}$. The first phase of the approximate algorithm is reporting the amount of needed or extra energy of each BS to the CCS and forming sets $\mathcal{S}$ and $\mathcal{B}$. In the second phase, like the nearest neighbor algorithm, the proposed algorithm starts from an arbitrary BS with energy deficit $b_{j}, \forall j \in \mathcal{B}$, and according to distances between BSs, it finds the closest BS with the extra harvested energy. Assume that this BS with the extra harvested energy is $s_{i}$. If $\eta_{b_{j}}^{\star}(t)$ is less than the extra stored energy in BS $s_{i}$ battery, $\rho_{s_{i}}(t)$, some amount of energy remains in the battery of the BS $s_{i}$ after $\eta_{b_{j}}^{\star}(t)$ Joule is transferred to the BS $b_{j}$. Next, the $\mathrm{BS} b_{j}$ is removed from $\mathcal{B}$. The extra energy of the BS $s_{i}$ is updated as $\rho_{s_{i}}(t)=\rho_{s_{i}}(t)-\eta_{b_{j}}^{\star}(t)$. If $\eta_{b_{j}}^{\star}(t)$ is more than the extra energy of the BS $s_{i}$, the total extra stored energy is shared with $b_{j}$. The extra energy of the BS $s_{i}$ goes to zero, $\rho_{s_{i}}(t)=0$. The BS $s_{i}$ is removed from $\mathcal{S}$. Additionally, the needed energy of the BS $b_{j}$ is updated as $\eta_{b_{j}}^{\star}(t)=\eta_{b_{j}}^{\star}(t)-\rho_{s_{i}}(t)$. The algorithm chooses the nearest BS with the extra stored energy for energy transfer to the BS $b_{j}$ until the BS $b_{j}$ acquires $\eta_{b_{j}}^{\star}(t)$ Joule. When the BS $b_{j}$ acquires $\eta_{b_{j}}^{\star}(t)$ Joule, the BS $b_{j}$ is removed from $\mathcal{B}$. Another BS is chosen from $\mathcal{B}$, and its energy deficit is compensated in the same way. The algorithm terminates when the set $\mathcal{B}$ is empty. The summary of the approximate algorithm for assigning the supply to the demand is given Table I. The proposed algorithm is run by the CCS which controls the network. Since the harvested energy by a BS is correlated in time, the high harvested energy in successive time slots culminates in waste of energy due to the BS limited battery capacity. As the extra stored energy of a BS is shared, the waste of energy reduces.

\section{Performance Results}

A three-tier small cell network is considered that includes macrocells (tier 1), microcells (tier 2) and picocells (tier 3). BSs of tiers are distributed according to PPPs with densities $\left[\frac{1}{500^{2}}, \frac{3}{500^{2}}, \frac{5}{500^{2}}\right] \mathrm{m}^{-2}$, respectively, in a $1.35 \mathrm{~km} \times 1.35 \mathrm{~km}$ area. Additionally, users distribution follows a PPP with density $\frac{80}{500^{2}} \mathrm{~m}^{-2}$. The transmit power of BSs, depending on their tier, sorted as $[40,6.3,1]$ Watt. The path-loss exponent is considered to be 4 , the coverage probability is 0.65 and the time slot duration is one second. BSs earn $\varphi=2500$ units of money by consuming one Joule to serve connected users. The nonrenewable energy consumption by a BS costs $\zeta=5000$ units of money per Joule. The cost of transferring one Joule by the smart grid is $\Gamma\left(g_{s_{i}, b_{j}}\right)=g_{s_{i}, b_{j}}$. The consumed power to serve each user is modeled as a correlated Gaussian process. The harvested energy by each BS is modeled as a correlated Gaussian process. Means of the demanded power from different tiers are $[22.5,5,0.85]$ Watt, respectively. Gaussian processes are correlated by Cholesky decomposition method. Both the harvested energy and the demanded energy at each time slot are correlated by their previous values at the last time slot and two previous time slot.

Fig. 1 depicts the effect of the proposed approximate algorithm and battery capacities on the consumed non-renewable power of tiers in 600 time slots. Means of the harvested energy of BSs in different tiers are $[23.3,5.8,1.1]$ Joule. The sum of the harvested energy of BSs in the network in all time slots are more than the demanded energy from BSs in the network. Thus, the harvested energy in BSs of the network is sufficient to serve connected users in all time slots. The harvested energy by a BS and the demanded energy are stochastic, therefore, the BS is with energy shortage in some time slots. By using the proposed approximate algorithm, the consumed power from the non-renewable source is reduced considerably. In the initial time slots, the larger battery capacity is not influential due to the fact that the extra stored energy is less than the battery capacity. As more time slots are elapsed, the extra stored energy increases, and the larger battery capacities become more helpful. The proposed algorithm distributes the extra stored energy among BSs with energy deficit instead of storing the extra energy in the batteries. Therefore, BSs require smaller batteries to store energy when the algorithm is applied. Applying the algorithm removes the cost of installing large batteries. When the algorithm 


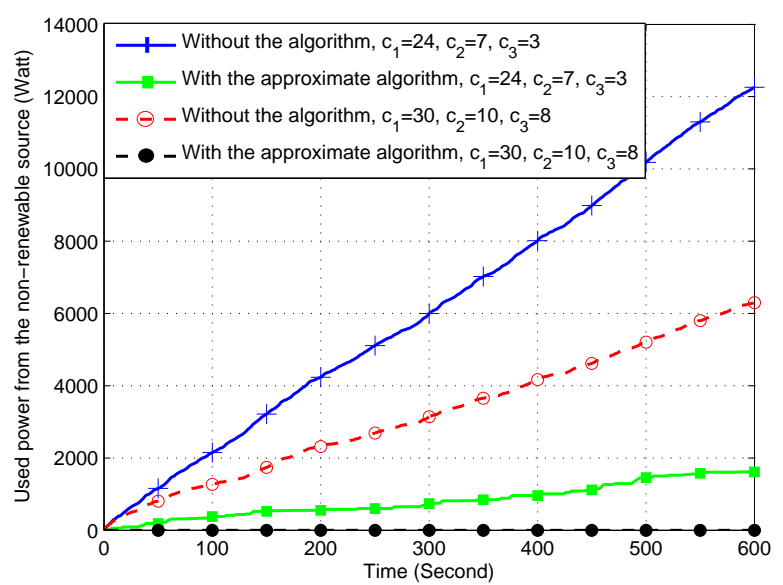

Fig. 1. Comparison of the non-renewable power consumption in the network with and without applying the approximate algorithm.

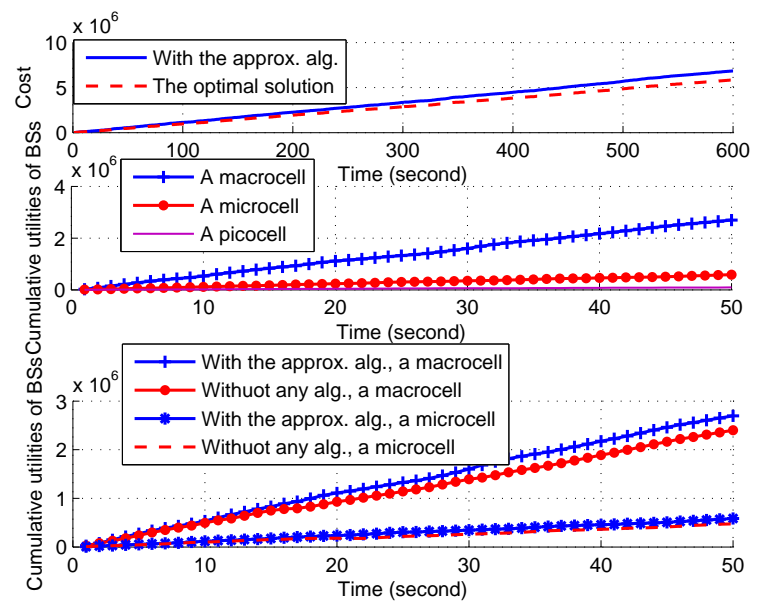

Fig. 2. Plot 1: the cost of the smart grid usage. Plot 2: cumulative utilities of a macrocell and a microcell when the approximate algorithm is applied. Plot 3: comparison of cumulative utilities of a macrocell and a microcell in the presence of the approximate algorithm and without it.

is not applied, the non-renewable energy consumption increases as the harvested energy is wasted in limited batteries.

The smart grid usage cost is depicted in Fig. 2. Reducing the smart grid usage cost increases the utility functions of BSs. Fig. 2 shows that the approximated solution found by the algorithm is near to the optimal solution. Since the algorithm distributes energy according to the closed-form of the energy share formula given in (9), the fairness of the energy distribution is one. The run time of the approximate algorithm for 600 time slots is 1.4 second. The cumulative utility functions of a macrocell, a microcell and a picocell when the approximate algorithm is applied are depicted in Fig. 2. As a BS consumes more energy, it earns more money. Hence, it is seen from Fig. 2 that a macrocell earns more revenue than BSs in the other tiers. The cumulative utilities of a macrocell and a microcell, in the presence of the approximate algorithm and without it, are depicted in Fig. 2 It is observed that when BSs share energy by the smart grid, BSs gain more utilities. Without the algorithm, the utilities of the BSs decrease by the high price of the non-renewable energy. However, with the algorithm, the non-renewable energy consumption reduces, and thus, the utilities of BSs increase. Hence, using the algorithm not only reduces the non-renewable energy consumption, it enhances BSs gained utilities as well.

\section{REFERENCES}

[1] J. G. Andrews, H. Claussen, M. Dohler, S. Rangan, and M. C. Reed, "Femtocells: Past, present, and future," IEEE J. Sel. Areas Commun., vol. 30, no. 3, pp. 497-508, Mar. 2012.

[2] T. Han and N. Ansari, "On greening cellular networks via multicell cooperation," IEEE Wireless Commun., vol. 20, no. 1, pp. 82-89, Mar. 2013.

[3] W. Tushar, S. Huang, C. Yuen, J. Zhang, D. B. Smith, et al., "Synthetic generation of solar states for smart grid: A multiple segment markov chain approach," in IEEE ISGT Europe, pp. 1-6, Oct. 2014.

[4] X. Chen, C. Yuen, and Z. Zhang, "Wireless energy and information transfer tradeoff for limited-feedback multiantenna systems with energy beamforming," IEEE Trans. Veh. Technol., vol. 63, no. 1, pp. 407-412, Jan. 2014.

[5] G. Auer, V. Giannini, C. Desset, I. Godor, P. Skillermark, M. Olsson, M. A. Imran, D. Sabella, M. J. Gonzalez, O. Blume, and A. Fehske, "How much energy is needed to run a wireless network?," IEEE Wireless Commun., vol. 18, no. 5, pp. 40-49, Oct. 2011.

[6] H. S. Dhillon, Y. Li, P. Nuggehalli, Z. Pi, and J. G. Andrews, "Fundamentals of heterogeneous cellular networks with energy harvesting," IEEE Trans. Wireless Commun., vol. 13, no. 5, pp. 2782 - 2797, May 2014.

[7] Y. Wang, W. Saad, Z. Han, H. V. Poor, and T. Basar, "A game-theoretic approach to energy trading in the smart grid," IEEE Trans. Smart Grid, vol. 5, no. 3, pp. 1439-1450, Apr. 2014.

[8] B. Gurakan, O. Ozel, J. Yang, and S. Ulukus, "Energy cooperation in energy harvesting communications," IEEE Trans. Wireless Commun., vol. 61, no. 12, pp. 4884-4898, Dec. 2013.

[9] Y.-K. Chia, S. Sun, and R. Zhang, "Energy cooperation in cellular networks with renewable powered base stations," IEEE Trans. Wireless Commun., vol. 32, no. 12, pp. 6996 - 7010, Dec. 2014.

[10] J. Xu, L. Duan, and R. Zhang, "Cost-aware green cellular networks with energy and communication cooperation," arXiv preprint arXiv:1411.4139, 2014.

[11] J. Xu and R. Zhang, "Cooperative energy trading in CoMP systems powered by smart grids," arXiv preprint arXiv:1403.5735, 2014.

[12] W. Tushar, B. Chai, C. Yuen, D. B. Smith, K. L. Wood, Z. Yang, and H. V. Poor, "Three-party energy management with distributed energy resources in smart grid," IEEE Trans. Ind. Electron., vol. 62, no. 4, pp. 2487-2498, Apr. 2015

[13] Y. Liu, C. Yuen, R. Yu, Y. Zhang, and S. Xie, "Queuing-based energy consumption management for heterogeneous residential demands in smart grid," May 2015.

[14] X. Jia, Q. Xia, and Q. Chen, "A new transmission cost allocation method considering power flow duration time in smart grid," in Proc. IEEE ISGT Europe, pp. 1-5, Oct. 2012.

[15] S. Zhong, J. Chen, and Y. R. Yang, "Sprite: A simple, cheat-proof, creditbased system for mobile ad-hoc networks," in Proc. IEEE Intl. Conf. on Comput. Commun. (INFOCOM), pp. 1987-1997, Mar. 2003.

[16] J. Lee and T. Q. Quek, "Hybrid full-/half-duplex system analysis in heterogeneous wireless networks," IEEE Trans. Wireless Commun., vol. 12, no. 99, Jan. 2013.

[17] J. Wong, J. Sauve, and J. Field, "A study of fairness in packet-switching networks," IEEE Wireless Commun., vol. 30, no. 2, pp. 346-353, Jan. 1982.

[18] H. J. Kushner and P. A. Whiting, "Convergence of proportional-fair sharing algorithms under general conditions," IEEE Trans. Wireless Commun., vol. 3, no. 4, pp. 1250-1259, July 2004.

[19] R. Jain, D.-M. Chiu, and W. R. Hawe, "A quantitative measure of fairness and discrimination for resource allocation in shared computer system," DEC Research Report DEC-TR-301, Sept. 1984.

[20] R. M. Freund, "Issues in non-convex optimization," [Online]. Available: http://ocw.mit.edu/courses/sloan-school-of-management/15-094j-systemsoptimization-models-and-computation-sma-5223-spring-2004/lecturenotes.

[21] N. Reyhanian, V. Shah-Mansouri, B. Maham, and C. Yuen, "Maximizing the fairness of energy distribution in cellular networks." [Online]. Available: $\quad$ https://docs.google.com/viewer?a=v\&pid=sites\&srcid= ZGVmYXVsdGRvbWFpbnxuYXZpZHJleWhhbmlhbnxneDo0NDk 4ZmI0MjYzMjIwZTkw

[22] D. S. Johnson and L. A. McGeoch, "The traveling salesman problem: A case study in local optimization," Local search in combinatorial optimization, vol. 1, pp. 215-310, 1997. 